\title{
Mixomicetos associados a coqueiro (Cocos nucifera L.) na Estação Experimental de Itapirema (Goiana, Pernambuco, Brasil)
}

\author{
Myxomycetes associated with coconut palm (Cocos nucifera L.) \\ at Itapirema Experimental Station (Goiana, Pernambuco, Brazil)
}

Camila Estelita Vogeley Alves de Sắ1* ${ }^{*}$ Luiz Gonzaga Biones Ferraz ${ }^{2}$, Laise de Holanda Cavalcanti

Universidade Federal de Pernambuco (UFPE), Av. Prof. Moraes Rego, 50670-901, Recife, PE, Brasil IInstituto Agronômico de Pernambuco (IPA), Recife, PE, Brasil

*autor correspondente \ camilavogeleysa@gmail.com
RESUMO: Cocos nucifera L. (Arecaceae) apresenta expressiva importância socioeconômica, graças aos diferentes produtos obtidos da planta. Os mixomicetos (Amoebozoa: Myxogastria) integram o grupo de organismos que comumente compõem a microbiota presente em coqueiros. Com o objetivo de ampliar o conhecimento sobre a mixobiota associada ao coqueiro, investigou-se a ocorrência de mixomicetos em coqueiral da Estação Experimental de Itapirema (Goiana, Pernambuco). Em seis excursões realizadas em diferentes estações do ano, avaliaram-se a incidência, a abundância e a constância das espécies em 100 coqueiros da variedade anã, nos seguintes substratos: estopa, bráctea floral (na planta e no solo); flor (folhedo aéreo); espigueta floral, bainha foliar e folíolo (na planta) e fruto caído no solo. Definiram-se os micro-hábitats preferenciais e a diversidade taxonômica da mixobiota. Exsicatas foram depositadas no Herbário da Universidade Federal de Pernambuco (UFP). Apresentam-se a descrição das espécies, os registros em palmeiras e a distribuição geográfica no Brasil. Mixomicetos foram encontrados em $57 \%$ dos coqueiros examinados e cinco espécies foram identificadas: Hemitrichia serpula (Scop.) Rostaf. ex Lister, Hemitrichia calyculata (Speg.) M. L. Farr; Physarella oblonga (Berk. \& M. A. Curtis) Morgan, Physarum stellatum (Massee) G. W. Martin e Physarum tenerum Rex. As espécies mais abundantes foram H. serpula $(80 \%)$ e P. stellatum (14\%); as demais são ocasionais. Hemitrichia serpula é uma espécie constante em C. nucifera; $H$. calyculata e $P$. stellatum são acessórias e $P$. oblonga e $P$. tenerum são acidentais. Dentre os micro-hábitats estudados, a estopa foi considerada o preferencial, por apresentar mais diversidade taxonômica $(1,33)$ e incidência de espécies (29\%).

PALAVRAS-CHAVE: Arecaceae, micro-hábitat, palmeira, Physaraceae, Trichiaceae.
ABSTRACT: Cocos nucifera L. (Arecaceae) presents significant socioeconomic importance, due to the different products obtained from the plant. The Myxomycetes (Amoebozoa: Myxogastria) are part of the organisms group, which composes the coconut palm microbiota. Concerning to the to increase the knowledge about coconut tree myxobiota, it was investigated the occurrence of Myxomycetes on coconut grove from Itapirema Experimental Station (Goiana, Pernambuco). In six excursions carried out in different seasons of the year, the incidence, abundance and constancy of the species were evaluated in 100 dwarf coconut, in the following substrates: tow, floral bract (on the plant and fallen to the ground), flower (aerial litter), inflorescence, leaf sheath and leaflets (on the plant), and fruit fallen to the ground. Preferred microhabitats and taxonomic diversity of myxobiota were defined. Exsiccates were deposited in the UFP Herbarium. Description of the species, records on palm trees and distribution in Brazil are presented. Myxomycetes were found in 57\% of the plants examined, and five species were identified: Hemitrichia serpula (Scop.) Rostaf. ex Lister, Hemitrichia calyculata (Speg.) M.L. Farr; Physarella oblonga (Berk. \& M.A. Curtis) Morgan, Physarum stellatum (Massee) G.W. Martin and Physarum tenerum Rex. The most abundant species were H. serpula (80\%) and P. stellatum (14\%); the others are occasional. Hemitrichia serpula is a constant species in C. nucifera; $H$. calyculata and P. stellatum are accessory and P. oblonga and P. tenerum are accidental. Among the studied microhabitats, the tow was considered the preferable one, because it had higher taxonomic diversity $(1,33)$ and higher incidence of species (29\%)

KEYWORDS: Arecaceae, microhabitat, palm tree, Physaraceae, Trichiaceae. 


\section{Introdução}

Cocos nucifera L. (Arecaceae), palmeira conhecida como "coqueiro" ou "coco-da-baía", é uma frutífera de expressiva importância socioeconômica mundial por oferecer vários produtos e subprodutos oriundos de diferentes partes da planta, principalmente dos frutos (LORENZI et al., 2010). O coqueiro também é bastante utilizado em projetos paisagísticos, com ênfase nas regiões tropicais (ARAGÃO et al., 2002). O Nordeste do Brasil é a região do país que mantém a maior participação na produção nacional de coco, segundo a Agência Embrapa de Informação Tecnológica (EMPRESA..., 2019).

Economicamente, são explorados mundialmente coqueiros de duas variedades (gigante e anã) e de híbridos intervarietais, oriundos do cruzamento entre elas. Por ser uma planta de crescimento indeterminado, o coqueiro somente cessa o aumento em altura quando entra em senescência. Representantes desses três grupos podem alcançar mais de 20 metros de altura, destacando-se o coqueiro gigante por ter crescimento mais rápido do que os dois e o híbrido mais do que o anão. $\mathrm{O}$ coqueiro anão é o mais precoce, podendo florescer a partir de dois anos de campo. Os frutos da variedade gigante e de híbridos são usados, preferencialmente, pela indústria alimentícia, enquanto os do anão o são para consumo de água.

Os cultivares de coqueiro são suscetíveis ao ataque de diversos agentes biológicos, os quais podem ocasionar danos à planta e prejuízos para produtores e comerciantes. As doenças mais comuns no Brasil, segundo a Ageitec, são: lixa-pequena e lixa-grande, causadas pelos fungos Camarotella torrendiella (Batista) Bezerra \& Vitória e Sphaerodothis acrocomiae (Mont.) Arx \& E. Müll.; queima das folhas, pelo agente Lasiodiplodia theobromae (Pat.) Griffon \& Maubl.; anel vermelho, pelo nematoide Bursaphelenchus cocophilus (Cobb) Baujard. Dentre as pragas, destaca-se, aqui, a broca do coqueiro, Rhynchophorus palmarum L. (Coleoptera: Curculionidae), que, em seu estado larval, se alimenta dos tecidos tenros da planta, formando extensas galerias no seu estipe. Na fase adulta, é transmissor do nematoide do anel vermelho e facilitador da entrada do fungo Thielaviopsis paradoxa (De Seynes) Hölh, causador da doença resinose-do-coqueiro. No filoplano do coqueiro estão presentes outros organismos, como os mixomicetos, que pertencem ao filo Amoebozoa, classe Myxogastria (FARR, 1960; PÔRTO; CAVALCANTI; CORREIA, 1985; DAGAMAC et al., 2017; MENDES; URBEN, 2019).

Os esporocarpos dos mixomicetos e os resíduos do plasmódio podem servir como locais de postura e fontes de alimento para coleópteros e outros insetos (HUYNH et al., 2017). Diferentes grupos de fungos atuam como saprófitos ou parasitas dos mixomicetos, na maioria das vezes inviabilizando a dispersão dos esporos (MUELLER; BILLS; FOSTER, 2004). Após a análise de esporocarpos provenientes de diferentes continentes, Rogerson e Stephenson (1993) identificaram nove espécies de Ascomycetes e 26 de Hyphomycetes (incluindo estados anamorfos e teleomorfos de um mesmo fungo), sendo Verticillium rexianum (Sacc.) Sacc. uma das mais frequentes. $\mathrm{Na}$ fase de metabolismo ativo, os mixomicetos fagocitam bactérias, fungos e protistas, mas também podem se alimentar diretamente por absorção de compostos orgânicos dissolvidos (STEPHENSON; ROJAS, 2017).

Os mixomicetos constituem um dos grupos mais ricos em espécies entre os protistas, capazes de sobreviver a condições ambientais adversas por meio de diferentes estratégias (esporulação, microcistos e macrocistos), e ocupam micro-hábitats existentes em ecossistemas bastante distintos, desde regiões superúmidas até desérticas (STEPHENSON; ROJAS, 2017). As palmeiras oferecem micro-hábitats favoráveis aos mixomicetos, como demonstrado por Pôrto, Cavalcanti e Correia (1985), que registraram 19 gêneros e 47 espécies em Cocos nucifera, Elaeis guineenses Jacq (dendezeiro) e Mauritia vinifera Mart. (buriti) no Brasil.

Esses organismos são pouco conhecidos no meio agronômico e Physarum cinereum (Batsch) Pers., por exemplo, por muito tempo, foi confundido com fungos dos gêneros Ustilago e Microbotryum (AGRA; SEIXAS; DIANESE, 2018). Existem registros de prejuízos causados por mixomicetos em cultivos de hortaliças, soja e cana de açúcar (AGRA; SEIXAS; DIANESE, 2018; MENDES; URBEN, 2019). Embora não sejam patógenos, a frequente coexistência de mixomicetos com insetos e fungos pode resultar em consequências negativas para o coqueiro, nos casos em que os esporocarpos possam ser utilizados como repositório para patógenos. Com o objetivo de ampliar o conhecimento sobre a incidência de mixomicetos em coqueiros e seus micro-hábitats preferenciais, realizou-se um inventário das espécies associadas a coqueiros tipo anão verde cultivados na Estação Experimental do Instituto Agronômico de Pernambuco, situada em Itapirema, município de Goiana, Pernambuco.

\section{Material e Métodos}

A Estação Experimental de Itapirema, do Instituto Agronômico de Pernambuco (EEI-IPA), está situada no município de Goiana $\left(7^{\circ} 33^{\prime} 46,85^{\prime \prime} \mathrm{S}\right.$ e $\left.35^{\circ} 00^{\prime} 47,31^{\prime \prime} \mathrm{O}\right)$, litoral norte de Pernambuco. Abrange uma área de 224 hectares, onde há plantios de coqueiros das variedades gigante e anã e de híbridos. O clima local é do tipo tropical úmido, com temperatura média anual em torno de $24{ }^{\circ} \mathrm{C}$, de julho a agosto, e de $27^{\circ} \mathrm{C}$, em fevereiro, com precipitação média anual em torno de $1.932,3 \mathrm{~mm}$, segundo a Companhia Pernambucana do Meio Ambiente (COMPANHIA..., 2003). As precipitações mensais aumentam a partir de janeiro, tendo o pico em junho e uma estação de estiagem de outubro a dezembro, com chuvas de outono-inverno, segundo o Climate Data (https://pt.climate-data.org). No período de outubro (começo da estiagem) a março, a temperatura máxima varia em torno de $30,5^{\circ} \mathrm{C}$, podendo chegar a $31,3{ }^{\circ} \mathrm{C}$ em janeiro, e as mínimas variam de $20,1^{\circ} \mathrm{C}$ a $21^{\circ} \mathrm{C}$. Entre abril e setembro se tem o período mais ameno, em que as temperaturas máximas variam de $28^{\circ} \mathrm{C}$ a $29^{\circ} \mathrm{C}$ e as mínimas ficam entre $18,6^{\circ} \mathrm{C}$ e 20,8 ${ }^{\circ} \mathrm{C}$ (CLIMATE DATA, 2019).

Foram realizadas seis excursões à EEI-IPA (março a maio de 2017; março e junho de 2018) para a coleta de esporocarpos e plasmódios em coqueiros adultos da variedade anã, tipo anão verde (dois a quatro metros de altura), tendo sido avaliados 10 a 20 indivíduos em cada ocasião (total $=100$ ), selecionados 
aleatoriamente. A busca por plasmódios e esporocarpos de mixomicetos foi efetuada nas seguintes partes das plantas: estopa, flor e bráctea floral (presas na planta ou caídas no solo), espigueta floral (presa na planta), bainha e folíolo (presos na planta) e fruto (caído ao solo). Para identificar os espécimes encontrados, foram utilizados os trabalhos de Farr (1976), Lado (2019) e Poulain, Meyer e Bozonnet (2011) e as exsicatas foram tombadas no Herbário da UFP. A distribuição no Brasil foi baseada em Cavalcanti, Agra e Bezerra (2019a, b).

A incidência dos mixomicetos nos coqueiros foi calculada pela razão entre o número de indivíduos em que foram encontrados esporocarpos e o número total de indivíduos examinados. O mesmo tipo de cálculo foi efetuado para avaliar a incidência nos diferentes substratos. Os valores foram expressos em porcentagem.

A abundância de cada espécie foi calculada segundo Schnittler e Stephenson (2000), baseada na distribuição proporcional entre o número de espécimes da espécie e o número total de espécimes de mixomicetos encontrados. As espécies foram classificadas como escassa (menos de 1,5\%), ocasional ( 1,5 a 3,5\%), comum (mais de 3,5 a 6,5\%) ou abundante (mais de 6,5\%).

A relação espécie/gênero (S/G) foi estimada para a mixobiota encontrada nos diferentes micro-hábitats. Dos valores obtidos pela relação, $\mathrm{S} / \mathrm{G}=1$ corresponde à máxima diversidade taxonômica, existindo uma espécie para cada gênero (DELA CRUZ et al., 2014). Sendo assim, quanto menor o valor da relação espécie/gênero, maior foi considerada a diversidade taxonômica no micro-hábitat.

A constância de cada espécie foi estimada relacionando o número de excursões realizadas e o número de excursões em que a espécie foi encontrada; as espécies foram classificadas como constante igual ou superior a $50 \%$, acessória igual a $25 \%$ a $50 \%$ ou acidental inferior ou igual a $25 \%$ (CAVALCANTI; MOBIN, 2004).

\section{Resultados e Discussão}

Inventário das espécies incidentes nos coqueiros

A incidência de mixomicetos no coqueiral da Estação Experimental de Itapirema foi de $57 \%$ para as plantas examinadas. Foram encontrados 64 espécimes na fase de esporocarpo e um na fase plasmodial, que não esporulou em laboratório. Cinco espécies foram identificadas, pertencentes às famílias Physaraceae (Physarella, Physarum) e Trichiaceae (Hemitrichia), descritas e comentadas a seguir.

Physarella oblonga (Berk. \& M.A. Curtis) Morgan, J. Cincinnati Soc. Nat. Hist. 19(1):7 (1896). UFP: 83.318 (Figura 1a).

Esporângio pedicelado, gregário, com 2,0 mm de altura. Esporoteca infundibuliforme, com 0,5 mm de diâmetro, amarelo-oliva. Pedicelo longo, cilíndrico, oco, castanho-avermelhado, translúcido. Hipotalo membranoso, rugoso na base do pedicelo. Perídio membranoso, amarelo-oliva, salpicado com pontos calcários amarelos; deiscência apical floriforme, os segmentos tornando-se reflexos e expondo os espinhos calcários amarelos, as paredes internas persistindo como uma pseudocolumela tubular e oca. Capilício abundante, composto de nódulos calcários amarelos em forma de espinho que se projetam das paredes internas do perídio e uma densa rede de filamentos delgados, hialinos, apresentando alguns nódulos calcários fusiformes, amarelos. Esporada castanho-escura. Esporo globoso, castanho-violeta sob luz transmitida, verruculoso, com $8 \mu \mathrm{m}$ de diâmetro.

Distribuição no Brasil: Norte (AM, PA, RR), Nordeste (AL, BA, PB, PE, PI, RN, SE), Centro-Oeste (DF), Sudeste (SP) e Sul (PR, RS, SC).

Registro em palmeiras no Brasil: Cocos nucifera L. (FARR, 1960; CAVALCANTI; SILVA, 1985), Elaeis guineensis Jacq. (RIBEIRO et al., 2003; SILVA; CAVALCANTI, 2010), Mauritia flexuosa L. f. (MOBIN; CAVALCANTI, 1998; CAVALCANTI; MOBIN, 2004).

Comentários: os esporângios de Physarella oblonga podem atingir até $3 \mathrm{~mm}$ de altura. $\mathrm{O}$ diâmetro da esporoteca atinge até $1 \mathrm{~mm}$, mas, em casos extremos, pode chegar a 1,4 mm, enquanto o diâmetro dos esporos varia de 6 a $9 \mu \mathrm{m}$. O único exemplar obtido situa-se no limite inferior de altura em relação ao tamanho dos esporocarpos, quando comparado aos descritos por outros autores que registraram sua presença em palmeiras no Brasil (FARR, 1960; CAVALCANTI; SILVA, 1985; CAVALCANTI; MOBIN, 2004; RIBEIRO et al., 2003; SILVA; CAVALCANTI, 2010).

Physarum stellatum (Massee) G. W. Martin, Mycologia 39 (4): 461 (1947). UFP: 82.856; 82.857; 82.861; 82.874; $82.877 ; 82.878 ; 82.879 ; 82.880 ; 82.865$ (Figura 1b).

Esporângio pedicelado, gregário, com $1,1 \mathrm{~mm}$ de altura, levemente inclinado. Esporoteca globosa, com $0,4 \mathrm{~mm}$ de diâmetro, cinza, umbilicada na base. Pedicelo preto na base, branco e calcário até o ápice. Hipotalo inconspícuo. Perídio fino, cinza, com placas de cálcio branco-leitoso na superfície, deiscência floriforme. Capilício delicado, esbranquiçado, com nódulos de cálcio normalmente formando uma pseudocolumela central. Esporada castanho-escura. Esporo globoso, castanho-claro, verruculoso, com $9 \mu \mathrm{m}$ de diâmetro.

Distribuição no Brasil: Norte (AM, AP, PA, RR), Nordeste (AL, BA, CE, PB, PE, PI, RN, SE), Centro-Oeste (DF, MT), Sudeste (RJ, SP) e Sul (PR, RS, SC).

Registro em palmeiras no Brasil: Mauritia flexuosa L. f. (MOBIN; CAVALCANTI, 1998; CAVALCANTI; MOBIN, 2004), palmeira não identificada (ALVES; CAVALCANTI, 1996; CAVALCANTI; PUTZKE, 1998).

Comentários: a altura total dos esporângios enquadrou-se no limite mínimo descrito para a espécie ( 1,0 a $1,3 \mathrm{~mm})$, assim como o diâmetro da esporoteca, que varia entre 0,4 e $0,6 \mathrm{~mm}$, enquanto os esporos apresentaram diâmetro no limite superior da faixa descrita para a espécie $(8$ a $9 \mu \mathrm{m})$. O material coletado nos coqueiros foi seguramente identificado como $P$. stellatum, apesar de alguns espécimes estarem mal formados.

Physarum tenerum Rex, Proc. Acad. Nat. Sci. Philadelphia 42:192 (1890). UFP: 84.436 (Figura 1c).

Esporocarpo pedicelado, gregário, com 1 a $3 \mathrm{~mm}$ de altura. Esporoteca globosa, com 0,3 a 0,4 mm de diâmetro, esverdeada, pendente. Pedicelo longo, com 0,7 a 2,5 mm de comprimento, subulado, amarelado no ápice e escurecendo gradativamente até a base, calcário. Columela ausente, mas a base do perídio apresenta um disco no ponto de encontro com o pedicelo. Perídio simples, membranoso, contendo cálcio de coloração 

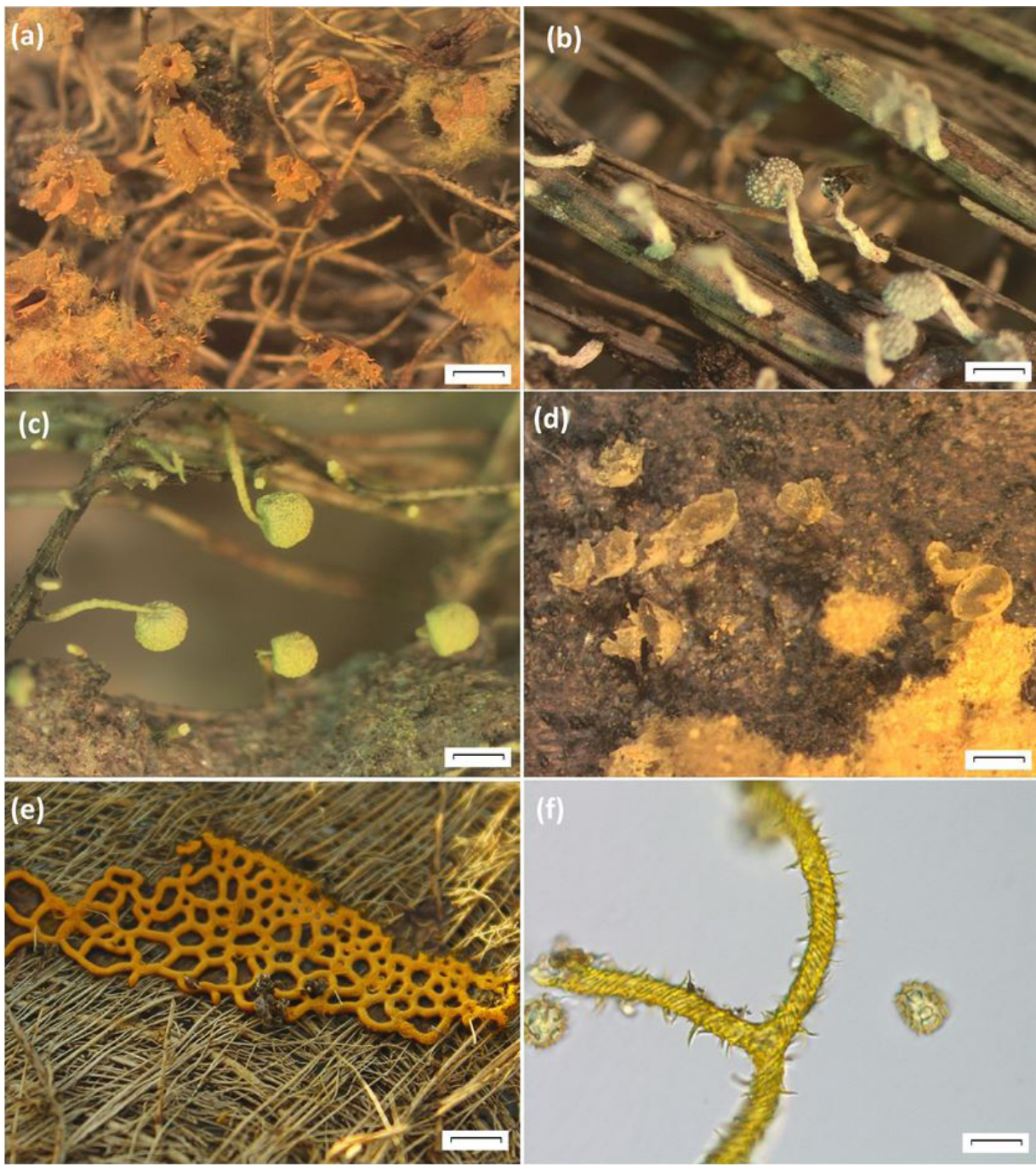

Figura 1. Espécies de mixomicetos encontradas em Cocos nucifera L. na Estação Experimental de Itapirema pertencente ao Instituto Agronômico de Pernambuco (Goiana, PE, Brasil). a) Esporângios de Physarella oblonga (1mm); b) Physarum stellatum $(500 \mu \mathrm{m})$; c) Physarum tenerum $(500 \mu \mathrm{m}) ; \mathrm{d})$ Hemitrichia calyculata (1mm); e) Plasmodiocarpo de Hemitrichia serpula; f) Capilício e esporos de Hemitrichia serpula $10 \mu \mathrm{m}$.

amarelo-esverdeada, deiscência floriforme. Capilício delicado, com nódulos de cálcio amarelados, pequenos, redondos, conectados por delicados filamentos hialinos. Esporada castanho-escura. Esporo globoso, violáceo, verrucoso, com $8 \mu \mathrm{m}$ de diâmetro.

Distribuição no Brasil: Norte (AM), Nordeste (BA, CE, PE, SE), Sudeste (SP) e Sul (RS).

Registro em palmeiras no Brasil: palmeira não identificada (HOCHGESAND; GOTTSBERGER, 1996).

Comentários: o espécime encontrado apresenta algumas características semelhantes a Physarum viride (Bull.) Pers., diferenciando-se pelo pedicelo calcário e pela ausência de columela. A base da esporoteca é achatada, não umbilicada, e forma uma espécie de "borda", característica não encontrada na descrição de $P$. tenerum (Figura 1c). Até o momento, a ocorrência dessa espécie em palmeira era conhecida apenas para o estado de São Paulo, constituindo a primeira referência para Cocos nucifera L.

Hemitrichia calyculata (Speg.) M. L. Farr, Mycologia 66 (5): 887 (1974). UFP: 83.319; 84.432 (Figura 1d).

Esporângio pedicelado, gregário. Esporoteca ovalada, com $1 \mathrm{~mm}$ de diâmetro (expandida), amarelada. Pedicelo longo, delgado, castanho-enegrecido, com 1,3 $\mathrm{mm}$ de comprimento. Hipotalo irregular. Perídio membranoso, amarelo, persistindo como um calículo após a deiscência. Capilício formado por uma densa rede amarelada de filamentos ornamentados com espirais. Esporada amarela. Esporo globoso, amarelo-pálido, verruculoso e levemente reticulado, com $8 \mu \mathrm{m}$ de diâmetro.

Distribuição no Brasil: Norte (AM, RR), Nordeste (AL, BA, CE, MA, PB, PE, PI, RN, SE), Centro-Oeste (DF), Sudeste (RJ, SP) e Sul (RS, SC).

Registro em palmeiras no Brasil: palmeira não identificada (CAVALCANTI; MARINHO, 1985; PÔRTO; CAVALCANTI; CORREIA, 1985; ALVES; CAVALCANTI, 1996), Attalea speciosa Mart. ex Spreng. (PARENTE; CAVALCANTI, 2013), Mauritia flexuosa L. f. (MOBIN; CAVALCANTI, 1998; CAVALCANTI; MOBIN, 2004), Astrocaryum vulgare Mart. (CAVALCANTI; MOBIN, 2004).

Comentários: os esporângios dos dois espécimes encontrados possuem altura total um pouco menor que a descrita por Martin e Alexopoulos (1969) para a espécie e por Bezerra, Farias e Cavalcanti (2010), para material coletado sobre palmeira no Brasil. 
Hemitrichia serpula (Scop.) Rostaf. ex Lister, Monogr. Mycetozoa, ed. 1, 179 (1894). UFP: 82.855;83.320);83.321; $83.323 ; 83.325 ; 84.419 ; 84.421 ; 82.859 ; 82.860 ; 84.422 ; 82.863$; $82.86 ; 82.867 ; 82.868 ; 84.435 ; 82.871 ; 82.872 ; 82.873 ; 82.875$; $84.442 ; 84.443 ; 84.444 ; 84.445 ; 82.876 ; 82.854 ; 82.858 ; 82.866$; $82.869 ; 84.425 ; 84.426 ; 84.427 ; 82.870 ; 83.322 ; 84.429 ; 84.437$; $84.438 ; 84.439 ; 84.440 ; 84.441 ; 84.430 ; 84.431 ; 84.433 ; 84.434$; 84.446 ; 83.324; 84. 420; 84.424; 84.423; 84.428; 82.862; 83. 326 (Figura 1e, f).

Plasmodiocarpo ramificado, reticulado, amarelo-ouro a amarelo-acastanhado. Perídio simples, membranoso, com deiscência longitudinal e irregular. Capilício ramificado, elástico, amarelo-ouro, com filamentos ornamentados com três a quatro bandas espirais, espinhos longos, com 3 a $5 \mu \mathrm{m}$ de comprimento (var. serpula) conectados por estrias longitudinais. Esporada amarelo-ouro. Esporo subgloboso, com reticulações largas e variáveis (var. serpula), amarelo-pálido sob a luz transmitida, com bordas de 1 a $2 \mu \mathrm{m}$ de espessura e 10 a $16 \mu \mathrm{m}$ de diâmetro.

Distribuição no Brasil: Norte (AM, AP, PA, RR), Nordeste (AL, BA, CE, PB, PE, PI, RN, SE), Centro-Oeste (DF, MT), Sudeste (RJ, SP) e Sul (PR, RS, SC).

Registro em palmeiras no Brasil: Astrocaryum vulgare Mart. (CAVALCANTI; MOBIN, 2004), Mauritia flexuosa L. f. (CAVALCANTI; MOBIN, 2004), Atallea speciosa Mart. ex. Spreng. (PARENTE; CAVALCANTI, 2013), Elaeis guineensis Jacq. (SILVA; CAVALCANTI, 2010), palmeiras não identificadas (FARR, 1985; PÔRTO; CAVALCANTI; CORREIA, 1985; CAVALCANTI; PUTZKE, 1998).

Comentários: em alguns espécimes, foram observadas variações na ornamentação do esporo visualizado sob aumento de x 1000, sem formar o retículo completo típico da espécie (Figura 1f). Foi constatada a presença de fungos em diversos espécimes, entretanto esse fato não comprometeu a identificação da espécie.

\section{Micro-hábitats e substratos ocupados}

Foram encontrados 51 espécimes de Hemitrichia serpula, nove espécimes de Physarum stellatum, dois espécimes de Hemitrichia calyculata e um único espécime de Physarella oblonga e de Physarum tenerum (Tabela 1). Em apenas um dos 100 coqueiros examinados foi coletado na estopa viva um faneroplasmódio, de espécie não identificada, porque não esporulou.
Considerando o material coletado na planta viva, os maiores percentuais de incidência nos coqueiros correspondem ao substrato oferecido pela estopa, seguido da bainha e da bráctea da inflorescência (Tabela 1). No folhedo sobre o solo em torno dos indivíduos examinados, não foram encontrados esporocarpos nem plasmódios de mixomicetos, e no folhedo aéreo, registrou-se apenas plasmodiocarpo de Hemitrichia serpula sobre restos florais.

A família Trichiaceae proporcionou o maior número de espécimes. Os plasmodiocarpos de Hemitrichia serpula apresentaram ampla distribuição nos substratos, não sendo registrados apenas no folíolo e no fruto, enquanto Hemitrichia calyculata foi encontrada apenas na bainha viva (Tabela 1). As três espécies de Physaraceae foram registradas na estopa viva e apenas Physarum stellatum esporulou também na bainha viva (Tabela 1).

Os mixomicetos são muito sensíveis às variações de temperatura e umidade nos seus micro-hábitats (STEPHENSON; ROJAS, 2017). Como referido por Dela Cruz et al. (2014), os ambientes tropicais, com elevada pluviosidade e temperaturas moderadas a quentes, somados à vegetação rica e diversa, resultam em uma elevada biodiversidade, onde se incluem os mixomicetos. Apesar de o clima de Goiana enquadrar-se no tipo tropical e de situar-se na Zona da Mata de Pernambuco, a monocultura do coqueiral e as características da arquitetura da palmeira estudada não correspondem ao esperado para a típica vegetação dos trópicos e as condições de luminosidade, ação dos ventos, balanço hídrico, entre outros fatores, não são das mais favoráveis ao desenvolvimento dos plasmódios dos mixomicetos. O inventário da mixobiota do coqueiral da EEI-IPA foi realizado no final da estiagem (março) e no período chuvoso (maio, junho), quando a umidade dos substratos é mais elevada e as temperaturas do ar são mais amenas. Nas coletas efetuadas em março, foram encontradas de uma a três espécies, representadas por dois a nove espécimes. Na estação chuvosa, o número de espécies foi semelhante, porém o de espécimes foi superior, chegando a 26 (H. serpula) em junho de 2018.

A falta de registro de esporocarpos no folhedo depositado no solo em torno dos coqueiros pode ser atribuída à lenta decomposição dos restos vegetais ocasionada pela baixa umidade, devida à rápida evaporação da água pela exposição aos raios solares e às correntes de vento, tornando esse micro-hábitat pouco propício ao estabelecimento dos mixomicetos.

Tabela 1. Substrato, abundância e constância das espécies de mixomicetos registradas em substratos oferecidos por Cocos nucifera L. na Estação Experimental de Itapirema - Instituto Agronômico de Pernambuco (Goiana, PE, Brasil).

\begin{tabular}{|c|c|c|c|}
\hline Espécie & Substrato/espécimes no & Abundância \% & Constância \% \\
\hline Physarella oblonga (Berk. \& M.A. Curtis) Morgan & Estopa $(\mathrm{V}) / 1$ & Ocasional 1,6 & Acidental 16,6 \\
\hline Physarum stellatum (Massee) G.W. Martin & $\begin{array}{l}\text { Estopa }(\mathrm{V}) / 8 \\
\text { Bainha }(\mathrm{V}) / 1\end{array}$ & Abundante 14,0 & Acessória 50,0 \\
\hline Physarum tenerum Rex & Estopa $(\mathrm{V}) / 1$ & Ocasional 1,6 & Acidental 16,6 \\
\hline Hemitrichia calyculata (Speg.) M.L. Farr & Bainha $(\mathrm{V}) / 2$ & Ocasional 3,1 & Acessória 33,3 \\
\hline Hemitrichia serpula (Scop.) Rostaf. ex Lister & $\begin{array}{c}\text { Estopa }(\mathrm{V}) / 24 \\
\text { Bainha }(\mathrm{V}) / 21 \\
\text { Bráctea }(\mathrm{V}) / 5 \\
\text { Flor }(\mathrm{M}) / 1\end{array}$ & Abundante 80,0 & Constante 100 \\
\hline
\end{tabular}

$\mathrm{V}=$ preso na planta viva; $\mathrm{M}=$ morto. 
O micro-hábitat oferecido pela estopa foi o preferencial para a maioria das espécies (Tabela 1), com um índice de diversidade taxonômica de 1,33. O micro-hábitat oferecido pela bainha viva apresentou menos diversidade taxonômica $(\mathrm{S} / \mathrm{G}=1,5)$, com três espécies de dois gêneros.

A diversidade taxonômica de mixomicetos registrados no coqueiral da EEI-IPA foi elevada $(\mathrm{S} / \mathrm{G}=1,67)$, muito próxima à referida por Mobin e Cavalcanti (2000) para a mixobiota de Copernicia prunifera (Miller) H. E. Moore, $(\mathrm{S} / \mathrm{G}=1,66)$, em cerrado do Piauí, mas não compartilham espécies. No estudo realizado em Teresina, também no Piauí, sobre as espécies associadas a indivíduos de Attalea speciosa Mart. ex Spreng. presentes em um fragmento de floresta mista subcaducifólia (PARENTE; CAVALCANTI, 2013), a diversidade taxonômica também foi elevada $(\mathrm{S} / \mathrm{G}=1,44)$, porém a composição da mixobiota apresenta baixa similaridade com a do coqueiro, tendo em comum apenas Hemitrichia calyculata e H. serpula.

Em ambiente de clima temperado na Nova Zelândia, empregando coletas no campo e cultivo em câmara-úmida, Stephenson (2003) registrou a presença de 37 espécies e 14 gêneros na bainha de folhas mortas da palmeira Rhopalostylis sapida $\mathrm{H}$. Wendl. \& Drude (Nikau). Apesar da maior riqueza de espécies, a diversidade taxonômica $(\mathrm{S} / \mathrm{G}=2,64)$ foi menor que a encontrada nos exemplares de $C$. nucifera examinados na EEI-IPA, tendo em comum apenas Hemitrichia serpula.

No Brasil, uma pesquisa realizada por Silva e Cavalcanti (2010) sobre a mixobiota de dendezeiros (Eleais guineenses Jacq.) presentes em um fragmento de Floresta Atlântica também evidenciou baixa diversidade taxonômica $(\mathrm{S} / \mathrm{G}=5,72)$, menor que a observada no coqueiral estudado, apesar da elevada riqueza de gêneros (11) e espécies (63), dentre elas $P$. oblonga e $H$. serpula, também registradas na EEI-IPA.

Hemitrichia serpula foi a mais abundante e constante dentre as espécies de mixomicetos presentes no coqueiral da EEI-IPA, particularmente na estação chuvosa, seguida de P. stellatum, abundante e acessória, sendo as demais classificadas como ocasionais e acessórias ou acidentais (Tabela 1).

Poucos espécimes foram encontrados na bráctea viva (5) e nos restos florais (1), todos representando Hemitrichia serpula; na bainha viva, foram registradas as duas espécies de Hemitrichia (23 espécimes) e Physarum stellatum (uma espécime), com $\mathrm{S} / \mathrm{G}=1,5$. A estopa viva evidenciou mais diversidade taxonômica e número de espécimes (34) que as observadas nos outros substratos, apresentando quatro gêneros e três espécies (Tabela 1).

Hemitrichia serpula foi constante no coqueiral da EEI-IPA, pois sua presença foi registrada em todas as coletas. Essa espécie frequentemente se associa com palmeiras, com registros de ocorrência em diferentes países, como no Brasil, Cuba, Costa Rica e Nova Zelândia (PÔRTO; CAVALCANTI; CORREIA, 1985; VILARÓ, 1991; SCHNITTLER; STEPHENSON, 2000; STEPHENSON, 2003; SILVA; CAVALCANTI, 2010).

\section{Conclusões}

Diferentes gêneros e espécies de mixomicetos estão associados aos coqueiros da EEI-IPA, esporulando em órgãos vivos, assim como nos restos vegetais ainda presos à planta-mãe.
A estopa viva é o substrato preferencial para a mixobiota do coqueiro.

Hemitrichia serpula foi considerada característica da mixobiota do coqueiral estudado.

\section{Referências}

AGRA, L. A. N. N.; SEIXAS, C. D. S.; DIANESE, J. C. False beans mut caused by slime mold. Plant Disease, Saint Paul, v. 102, n. 3, p. 507-510, 2018. http://dx.doi.org/10.1094/PDIS-06-17-0831-RE.

ALVES, M. H.; CAVALCANTI, L. H. Myxomycetes em palmeiras (Arecaceae). Acta Botanica Brasilica, Feira de Santana, v. 17, n. 1, p. 1-7, 1996.

ARAGÃO, W. M. et al. Florescimento, produção e composição morfológica de frutos de cultivares de coqueiro. Agrotropica, Ilhéus, v. 14, n. 3, p. 151-158, 2002

BEZERRA, M. F.A.; FARIAS, G. R.; CAVALCANTI, L. H. Mixobiota do Parque Nacional Serra de Itabaiana, SE, Brasil: Trichiales. Acta Botanica Brasilica, São Paulo, v. 24, n. 2, p. 510-517, 2010.

CAVAlCANTI, L. H.; AGRA, L. A. N. N.; BEZERRA, A. C. C. Physarales. In: JARDIM BOTÂNICO DO RIO DE JANEIRO - JBRJ. Flora do Brasil 2020 em construção. Rio de Janeiro, 2019a. Disponível em: $<$ http://floradobrasil.jbrj.gov.br/reflora/floradobrasil/ FB95345>. Acesso em: 21 jan. 2019.

CAVAlCANTI, L. H.; AGRA, L. A. N. N.; BEZERRA, A. C. C. Trichiales. In: JARDIM BOTÂNICO DO RIO DE JANEIRO - JBRJ. Flora do Brasil 2020 em construção. Rio de Janeiro, 2019b. Disponível em: $<$ http://floradobrasil.jbrj.gov.br/reflora/floradobrasil/ FB95345>. Acesso em: 21 jan. 2019.

CAVALCANTI, L. H.; MARINHO, M. G. V. Myxomycetes da Paraíba I - Trichiales. In: REUNIÃO NORDESTINA DE BOTÂNICA, 8., 1985, Recife. Anais... Recife: Sociedade Botânica do Brasil, Seccional Pernambuco, 1985. p. 185-191.

CAVALCANTI, L. H.; MOBIN, M. Myxomycetes associated with palm trees at Sete Cidades National Park. Systematic and Geography of Plants, Meise, v. 74, p. 109-127, 2004.

CAVALCANTI, L. H.; PUTZKE, J. Myxomycetes da Chapada do Araripe (Crato - CE, Brasil). Acta Botanica Brasilica, Feira de Santana, v. 12, n. 3, p. 253-261, 1998.

CAVAlCANTI, L. H.; SILVA, J. V. B. Myxomycetes da Paraíba III. Physarales. In: REUNIÃO NORDESTINA DE BOTÂNICA, 8., 1985, Recife. Anais... Recife: Sociedade Botânica do Brasil, Seccional Pernambuco, 1985. p. 199-205.

CLIMATE DATA. 2019. Disponível em: <https://pt.climate-data. org >. Acesso em: 16 fev. 2019.

COMPANHIA PERNAMBUCANA DO MEIO AMBIENTE - CPRH. Diagnóstico socioambiental do litoral norte de Pernambuco. Recife, 2003. 32 p.

DAGAMAC, N. H. A. et al. Speciation in progress? A phylogeographic study among populations of Hemitrichia serpula (Myxomycetes). PLoS One, San Francisco, v. 12, n. 4, p. 22, 2017.

DELA CRUZ, T. E. E. et al. A comparative species listing of myxomycetes from tropical (Philippines) and temperate (United States) forests. Mycosphere, Guiyang, v. 5, n. 2, p. 299-311, 2014.

EMPRESA BRASILEIRA DE PESQUISA AGROPECUÁRIA EMBRAPA. Agência Embrapa de Informação Tecnológica -AGEITEC. 2019. Disponível em: <https://www.agencia.cnptia. embrapa.br>. Acesso em: 10 fev. 2019. 
FARR, M. L. The Myxomycetes of the IMUR herbarium with special reference to Brazilian species. Recife: Universidade do Recife, 1960. p. 3-54. (Publicação, 184).

FARR, M. L. Myxomycetes. New York: The New York Botanical Garden, 1976. (Flora Neotropica. Monografia, 16).

FARR, M. L. Notes on Myxomycetes. IV. species collected in Brazil and Japan. Nova Hedwigia, Berlin, v. 41, p. 167-175, 1985.

HOCHGESAND, E.; GOTTSBERGER, G. Myxomycetes from state of São Paulo, Brazil. Boletim do Instituto de Botânica, São Paulo, v. 10, p. 1-47, 1996.

HUYNH, T. T. M. et al. Biological activities and chemical compositions of slime tracks and crude exopolysaccharides isolated from plasmodia of Physarum polycephalum and Physarella oblonga. BMC Biotechnology, London, v. 17, n. 1, p. 76, 2017.

LADO, C. An on-line nomenclatural information system of Eumycetozoa. 2019. Disponível em: <www.nomen.eumycetozoa. com>. Acesso em: 16 fev. 2019.

LORENZI, H. et al. Flora brasileira: Arecaceae (palmeiras). Nova Odessa: Plantarum, 2010. 384 p.

MARTIN, G. W.; ALEXOPOUlOS, C. J. The Myxomycetes. Iowa City: University of Iowa Press, 1969. 561 p.

MENDES, M. A. S.; URBEN, A. F. Fungos relatados em plantas no Brasil. Brasília: Laboratório de Quarentena Vegetal, Embrapa Recursos Genéticos e Biotecnologia, 2019. Disponível em: $<$ http// pragawall.cenargen.embrapa.br/arqweb/michtml/fgbanco01.asp $>$. Acesso em: 16 fev. 2019.

MOBIN, M.; CAVALCANTI, L. H. Myxomycetes ocorrentes sobre buritti (Mauritia flexuosa L. f., Arecaceae). Revista da Universidade do Amazonas Série Ciências Biológicas, Manaus, v. 2-3, p. 43-51, 1998.

MOBIN, M.; CAVALCANTI, L. H. Myxomycetes em Carnaubeira (Copernicia prunifera (Miller) H. E. Moore, Arecaceae). Acta Botanica Brasilica, Feira de Santana, v. 14, n. 1, p. 71-75, 2000.
MUELLER, G. M.; BILLS, G. F.; FOSTER, M. S. (Org.). Biodiversity of Fungi: inventory and monitoring methods. Burlington: Elsevier Academic Press, 2004. 777 p.

PARENTE, M. P. M.; CAVALCANTI, L. H. Myxomycetes on palm trees: species on Attalea speciosa Mart. ex Spreng. Advances in Microbiology, Irvine, v. 3, n. 8, p. 19-23, 2013.

PÔRTO, K. C.; CAVALCANTI, L. H.; CORREIA, M. A. S. Incidência de Myxomycetes em Palmae. In: CONGRESSO NACIONAL DE BOTÂNICA, 33., 1982, Maceió. Anais... Maceio: Sociedade Botânica do Brasil, 1985. p. 181-187.

POULAIN, M.; MEYER, M.; BOZONNET, J. Les Myxomycètes. Sevrier: Fédération Mycologique et Botanique Dauphiné-Savoie, 2011. v. 2.

RIBEIRO, S. M. et al. Plasmodium immobilization of Physarella oblonga (Berk. \& Curt.) Morgan (Myxomycetes) using kaolinite as a matrix of entrapment. Research in Microbiology, Paris, v. 154, n. 1, p. 55-58, 2003.

ROGERSON, C. T.; STEPHENSON, S. L. Myxomyceticolous fungi. Mycologia, Lancaster, v. 85, n. 3, p. 456-469, 1993.

SCHNITTLER, M.; STEPHENSON, S. L. Myxomycetes biodiversity in four different forest types in Costa Rica. Mycologia, Madinson, v. 92 , n. 4, p. 626-637, 2000. http://dx.doi.org/10.1080/00275514 .2000 .12061203 .

SILVA, C. F.; CAVALCANTI, L. H. Myxobiota of the Brazilian Atlantic Forest: species on oil palm tree (Elaeis guineensis, Arecaceae). Rodriguésia, Rio de Janeiro, v. 61, n. 4, p. 575-583, 2010.

STEPHENSON, S. L. Myxomycetes associated with decaying fronds of nikau palm (Rhopalostylis sapida) in New Zealand. New Zealand Journal of Botany, Wellington, v. 41, n. 2, p. 311-317, 2003.

STEPHENSON, S. L.; ROJAS, C. Myxomycetes: biology, systematics, biogeography, and ecology. London: Academic Press, 2017. 476 p.

VILARÓ, M. Myxomycetes de Cuba. Revista Jardin Botánico Nacional, Havana, v. 12, p. 127-131, 1991.

Recebido: 06 set. 2019 Aprovado: 06 nov. 2019 